\title{
DERECHOEIECTORAL
}

\section{Representatividad de minorías indígenas en América Latina}

Francinie Cubero de la Vega*

DOI 10.35242/RDE_2020_29_10

\section{Nota del Consejo Editorial}

Recepción: 30 de setiembre de 2019.

Revisión, corrección y aprobación: 15 de noviembre de 2019.

Resumen: La Declaración de los Derechos Humanos establece la prohibición de discriminación por etnia, raza, religión o cultura; sin embrago, las minorías se encuentran en desventaja política al verse imposibilitadas de acceder a puestos de poder. El desarrollo democrático ha permitido que, por reformas legales y constitucionales, se asegure la representatividad de minorías étnicas en el congreso por medio de la asignación de escaños. En América Latina, existen países que fijan escaños para estas minorías, el presente artículo pretende comparar a Colombia, Venezuela y Bolivia en el manejo de la representación de minorías. No se deja de lado el estudio de otros países con importante población étnica y la determinación de si permiten o no la representación de minorías.

Palabras clave: Representación política / Cuotas de participación política / Sistema de elección de diputados / Minoría legislativa / Partidos minoritarios / Grupos minoritarios / Grupos étnicos / Legislación electoral comparada / Derechos políticos / Colombia / Venezuela / Bolivia / América Latina.

Abstract: The Declaration of Human Rights establishes the prohibition of discrimination based on ethnicity, race, religion or culture. Minorities are at a political disadvantage when they are unable to access positions of power. Democratic development has allowed legal and constitutional reforms to ensure the representation of ethnic minorities in congress through the allocation of seats. In Latin America, there are countries that set aside seats for these minorities. This paper will compare Colombia, Venezuela and Bolivia in the management of minority representation. The article also includes the study of other countries with considerable ethnic population and whether they allow minority representation.

Key Words: Political representation / Political representation quotes / System of election of congress members / Legislative minority / Minority parties / Minority groups / Ethnic groups / Comparative electoral legislation / Political rights / Colombia / Venezuela / Bolivia / Latin America.

\footnotetext{
* Costarricense, abogada. Correo francinie@gmail.com. Licenciada en derecho. Especialista en derecho notarial y registral y máster en Gerencia de Proyectos por ULACIT; mediadora y conciliadora certificada por el CAM del Colegio de Abogados y Abogadas de Costa Rica, profesora universitaria, actualmente abogada asistente de la Procuraduría General de la República en Área de la Función Pública, con amplia experiencia en instrucción de procedimientos administrativos.
} 


\section{DERECHO ELECTORAL}

\section{INTRODUCCIÓN}

El presente artículo tiene como propósito realizar una comparación entre tres países latinoamericanos con características similares entre sí en cuanto a ubicación, conformación y que cuenten con población étnica de relevancia según los datos obtenidos y las leyes especiales. Al momento de elegir los países, se consideró que el mecanismo de elección sea democrático, con voto directo enfocando el estudio en la elección de miembros del congreso. Los países elegidos fueron Colombia, Bolivia y Venezuela; sin embargo, no se omite la referencia a otros países que se consideraron relevantes para el tema.

El estudio se centró en estudiar cada país y en determinar si cuentan con mecanismos a nivel constitucional y/o legal que permitan identificar si refieren con una norma que garantice la representación de minorías, realizando el enfoque en las minorías étnicas únicamente. Una vez acreditado esto, se analiza la forma en la que son elegidos, si tienen o no una circunscripción especial, además de si existen requisitos especiales por considerar para poder participar y la cantidad de escaños fijos que se ofrecen o no.

Con la información recolectada, se procederá a realizar una tabla comparativa de los países referidos para visualizar la información de una manera fácil y práctica que permita identificar cómo, en la realidad, es aplicable la representación de minorías étnicas.

\section{CONSIDERACIONES GENERALES DE MINORÍAS ÉtNICAS Y SU RELEVANCIA EN EL Derecho InTERnACIONAL}

La Declaración de las Naciones Unidas sobre las Minorías señala la necesidad de contar con factores objetivos (como la existencia de una etnia, de un lenguaje o de una religión compartidos) y factores subjetivos de las personas para identificarse a sí mismas como miembros de una minoría (ACNUDH, 2010).

Según la definición dada en 1977 por Francesco Capotorti, relator especial de la Subcomisión de Prevención de Discriminaciones y Protección a las Minorías y citado por la Oficina del Alto Comisionado para los Derechos Humanos (ACNUDH), una minoría es: 


\section{DERECHO ELECTORAL}

Un grupo numéricamente inferior al resto de la población de un Estado, que se encuentra en una posición no dominante y cuyos miembros, que son nacionales del Estado, poseen características étnicas, religiosas o lingüísticas diferentes de las del resto de la población y manifiestan, aunque sólo sea implícitamente, un sentimiento de solidaridad para preservar su cultura, sus tradiciones, su religión o su idioma (2010, p. 3).

En ese orden de ideas Barth considera que para que un grupo minoritario sea considerado étnico debe: 1) autoperpetuarse biológicamente; 2) compartir valores culturales fundamentales; 3 ) crear un campo propio de comunicación e interacción; 4) la pertenencia al grupo es identificable como tal por otros (1976, pp. 2-3).

Los distintos convenios internacionales en procura de los derechos de minorías en general y de grupos étnicos en específico ${ }^{1}$ buscan las aplicaciones de principios de igualdad y de no discriminación; para Flores (2014), la prohibición de cualquier discriminación entre los ciudadanos por motivos de origen étnico es una característica esencial de todo Estado democrático y de derecho, por lo que es un instrumento clave para la estabilidad política y la cohesión social de los Estados donde este pluralismo existe.

Como parte de los desafíos referidos por Flores (2014) que se dieron en Europa en cuanto a la participación de minorías étnicas en la representación parlamentaria, se identificaron cinco grupos de enfoques que se podían encontrar en las leyes electorales de acuerdo con las siguientes estrategias:

- Oponerse a la representación parlamentaria de las minorías étnicas: Conlleva un impedimento total de representación de minorías, o bien, la implementación de legislación electoral con reglas dirigidas a hacer más difícil o imposible la representación parlamentaria.

- Obviar la cuestión de la representación parlamentaria de las minorías étnicas: no realizar excepciones en leyes electorales al principio

\footnotetext{
1 Ejemplo: Convención relativa a la Lucha contra las Discriminaciones en la Esfera de la Enseñanza 1960, Convención Internacional sobre la Eliminación de la Discriminación Racial de todas las Formas de Discriminación Racial, Declaración final y Plan de Acción de la Tercera Conferencia Mundial contra el Racismo, la Discriminación Racial, la Xenofobia y las Formas Conexas de Intolerancia, celebrada en Durban, Sur África en el año 2001; Convenio relativo a la protección e integración de las poblaciones indígenas y de otras poblaciones tribales y semitribales en los países 1957 (OIT, Convenio 107), Convenio sobre Pueblos Indígenas y Tribales 1989 (OIT, Convenio 169), Declaración de las Naciones Unidas sobre los derechos de los Pueblos Indígenas 2007, Declaración Americana sobre Derechos de los Pueblos Indígenas OEA 2016
} 


\section{DERECHO ELECTORAL}

constitucional de igualdad y no discriminación, por no contar con minorías étnicas o porque esas tienen un peso demográfico importante.

- Facilitar la representación parlamentaria de las minorías étnicas: por medio de la legislación electoral se incluyen cláusulas o artículos encaminados para facilitar, mas no asegurar, la presencia de minorías étnicas en el Parlamento; esto por medio de excepción de requisitos en candidaturas o normas especiales en la asignación de escaños.

- Garantizar la representación parlamentaria de las minorías étnicas: la legislación electoral, o bien, la Constitución garantiza expresamente a las minorías étnicas un número mínimo de escaños. Esto se puede ver fácilmente reflejado en la creación de circunscripciones electorales especiales, o por medio de la forma de designación de escaños según la votación.

- Hacer de la adscripción étnica la base de la representación política: la distribución proporcional de los escaños se realiza en función de las etnias por lo que pueden ser considerados "etnocracia".

Tal y como se procederá a analizar, la anterior división puede ser evidenciada en los países de América Latina que se estudian, donde se evidenciarán el grado de actividad, participación y representación de minorías étnicas en los diferentes congresos, y la normativa brindada al efecto.

\section{ReVisión de nORMATIVA Legal Y CONSTitucional de los países SELECCIONADOS}

En el siguiente acápite, se procederá a estudiar de forma individual los países en los que se basará la comparación. Como primer elemento, se revisará si la Constitución Política de cada país hace referencia en forma alguna a la representación de minorías en el órgano legislativo, de igual manera, en caso de hacer referencia, se procederá a revisar la normativa legal.

Como segundo aspecto, se identificará la forma en que se regula dicha participación (circunscripciones especiales, requisitos de participación, etc.) para, finalmente, identificar en la doctrina aspectos importantes respecto de cada país que puedan brindar una idea general de la correcta aplicación o no de los mecanismos de representatividad. 


\section{DERECHOELECTORAL}

\section{a) Colombia}

"La Constitución de 1991 definió que las comunidades indígenas de Colombia debían estar representadas por dos miembros en el Senado de la República y un miembro en la Cámara de Representantes, con el objetivo de tener participación política y legislativa en el Congreso" (Colprensa, 2018, párr. 2).

El artículo 176 de la Constitución Colombiana, establece el mecanismo por el cual se elige a la Cámara de Representantes; cada departamento y el distrito capital de Bogotá conforman una circunscripción territorial con dos representantes por cada circunscripción territorial y uno más por cada 365 000 habitantes y fracción en exceso mayor de 182500 . Se establece un representante adicional para la circunscripción territorial conformada por el departamento de San Andrés, Providencia y Santa Catalina, que corresponderá a un representante por la comunidad raizal ${ }^{2}$.

De igual manera, se establecen circunscripciones especiales que aseguraran la participación en la Cámara de Representantes de los grupos étnicos y de los colombianos residentes en el exterior. Dichas circunscripciones se dividen en: comunidades afrodescendientes (2 representantes), comunidades indígenas (un representante), e internacional (un representante).

Por su parte, la Ley 649 del 27 de marzo de 2001 y que reglamenta el artículo 176 de la Constitución Colombiana, respecto de las circunscripciones especiales, en su artículo 2 establece que los candidatos de las comunidades indígenas que aspiren a ser elegidos a la Cámara de Representantes en dicha circunscripción deben acreditar con certificado de organización indígena, refrendado por el Ministerio del Interior, que han ejercido un cargo de autoridad tradicional en su respectiva comunidad o han sido líderes de una organización indígena.

De igual manera, para el caso de la circunscripción especial de comunidad negra, el artículo 3 establece que los candidatos deberán ser miembros de la respectiva comunidad y avalados previamente por una organización

\footnotetext{
${ }^{2}$ Los raizales son una comunidad étnica, porque tienen su propia lengua y cultura desarrollada a partir de sus raíces africanas, europeas y caribeñas. Sus raíces culturales afro-anglo-antillanas se manifiestan en una fuerte identidad cultural que se diferencia del resto de la población colombiana (Universidad del Rosario, 2019).
} 


\section{DERECHOELECTORAL}

inscrita ante la Dirección de Asuntos de Comunidades Negras del Ministerio del Interior.

Se llama la atención que, en el caso colombiano, existe una representación para minorías políticas en cuya circunscripción especial podrán participar los movimientos o partidos políticos:

a) Que hubiesen presentado candidatos a la Cámara de Representantes como mínimo en un 30\% de las circunscripciones territoriales; b) Que no hubiesen obtenido representantes en el Congreso Nacional, y c) Que su votación mayoritaria en un mismo departamento o circunscripción territorial sea menos del $70 \%$ de la sumatoria de su votación en todo el país. La curul corresponderá al partido o movimiento político que, cumpliendo con los requisitos, de los literales anteriores obtenga la mayor votación agregada en todo el país. La lista a la cual se le asignará la curul será la conformada por las cabezas de lista de mayor a menor votación de las inscritas por el respectivo partido o movimiento en todo el territorio nacional. (Art. 4, Ley 649, 2001).

Dentro de las regulaciones generales que establece dicha ley, los representantes a la Cámara por la circunscripción especial serán elegidos mediante el sistema que en el momento sirva de escogencia a los congresistas; y se establece que ninguna persona podrá votar simultáneamente por un candidato a la Cámara de circunscripción territorial y por un candidato a la Cámara de circunscripción especial.

Esta circunstancia permite que no solo los miembros de las comunidades indígenas o afroamericanas tengan la posibilidad de elegir a quiénes los van a representar en el Congreso, sino que cualquier ciudadano pueda votar, si lo desea, en la casilla de la circunscripción especial indígena, según lo establecido en el artículo 6 de la citada ley.

Según los datos recabados del Censo del 2018 por parte del Departamento Administrativo Nacional de Estadísticas (DANE) de Colombia, para el año 2018 la población a nivel nacional correspondería a 48258 494, de los cuales $1905617(4,4 \%)$ corresponde a población que se auto reconoce como indígena, como lo ilustra la figura 1 ; sin embargo, esta cantidad es mayor, ya que no se contabilizó a los pueblos indigenas Jurumi, Passe y Yuri en respeto de su aislamiento voluntario. 


\section{DERECHO ELECTORAL}

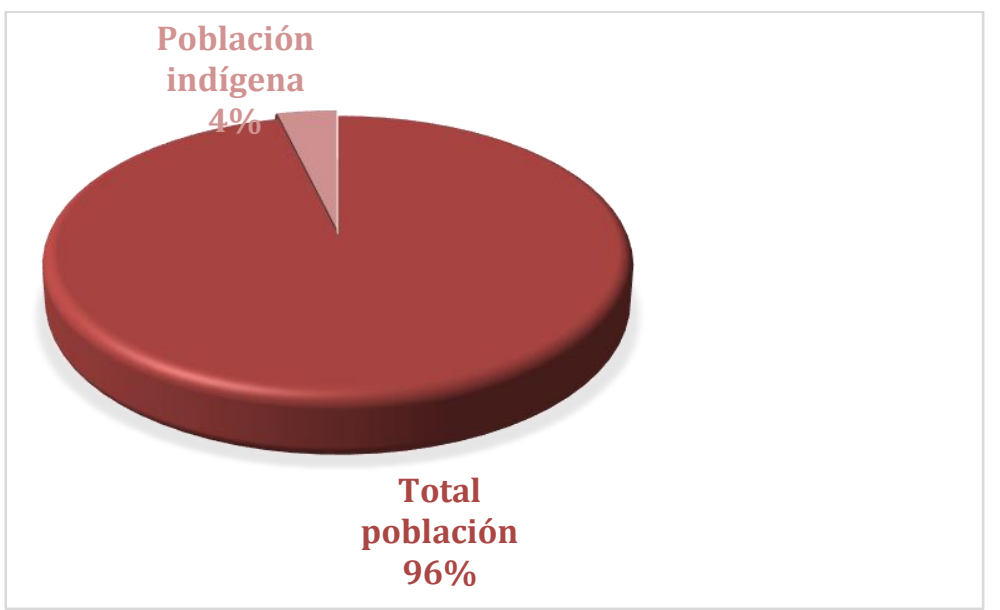

Figura 1. Porcentaje de población a nivel nacional, Colombia. Elaborado con base en los datos del censo de 2018, Departamento Administrativo Nacional de Estadísticas.

Ahora bien, la Cámara de Representantes de Colombia se compone de 172 legisladores (Cámara de Representantes, 2019), de los cuales y según la cantidad de población de acuerdo al Censo 2018, cada legislador representa a 280572 colombianos; en de vista que la Cámara de Representantes solamente tiene un miembro de representación indígena, para la cantidad de población indígena (1 905 617), debería corresponderle al menos 6 representantes de la Cámara. Esto quiere decir, que el representante indígena representa el $1,72 \%$ de la población, cuando la población indígena representa un $4,4 \%$.

Para Herrán (2009), la participación de minorías indígenas reconoce las luchas de las organizaciones indígenas al reordenamiento territorial de sus comunidades y el fortalecimiento de las autoridades tradicionales; refiere que con la Constitución Política de 1991 pasó de una constitución homogenizadora a una que definió como ideal la riqueza de la diversidad étnica y cultural, cambiando con ello el concepto de igualdad formal para dar paso a una igualdad material o real.

Por su parte, la Registraduría Nacional del Estado Civil de Colombia (s.f.) realizó un estudio respecto de la representación, participación y curules especiales de minorías étnicas, en ella concluye que la discusión sobre el tema tiene antecedentes europeos y norteamericanos en la que buscaron como solución la creación de mecanismos normativos de discriminación positiva concebidos para permitir la representación de diferentes grupos 


\section{DERECHO ELECTORAL}

sociales, generalmente discriminados por razones de raza, lengua, género o religión.

Por otra parte, la Corte Constitucional de Colombia en la sentencia C-702/10 analiza que el establecimiento de representación política de minorías sociales por medio de circunscripciones electorales especiales tenía como objetivo mejorar la representatividad del Congreso de la República y reconocer y proteger la diversidad étnica y cultural de la nación colombiana; sin embargo, el establecimiento de las circunscripciones especiales no es suficiente para asegurar una mejor representación ni resolver las principales desigualdades sociales.

Con base en los resultados de su estudio, y en atención a las circunscripciones especiales, la Registraduría refiere que candidatos indígenas 0 negros pueden lograr curules más fácilmente en circunscripciones departamentales ordinarias que en las circunscripciones especiales para la Cámara, por lo cual, la población no se motiva para a participar en el marco de las circunscripciones especiales; ello refleja más que una fragmentación política, una fragmentación social.

\section{b) BoLIVIA}

La Constitución Política Boliviana establece el reconocimiento precolonial de las naciones y pueblos indígenas originarios campesinos; con ello, su artículo 26 indica que los pueblos originarios (así referidos a pueblos indígenas), gozan del derecho de participación política, su inciso 2) señala: "(...) 4. La elección, designación y nominación directa de los representantes de las naciones y pueblos indígenas originario campesinos, de acuerdo con sus normas y procedimientos propios $(\ldots)^{\prime \prime}$.

Para ello, el cuerpo constitucional cuenta con las circunscripciones especiales indígena originario campesinas, en el que se establece que se regirán por el principio de densidad poblacional en cada departamento, ubicados únicamente en el área rural y en aquellos departamentos en que constituyan una minoría poblacional; dicha determinación está delegada en el órgano electoral, la cual refiere a lo establecido en el artículo 4 de Constitución Política.

En razón de ello, la Ley de Régimen Electoral establece una serie de principios de democracia intercultural sobre la cual resaltan la importancia 


\section{DERECHO EIECTORAL}

y existencia de los pueblos originarios, así como los afrobolivianos; el artículo 2 inciso J) establece que "El régimen electoral boliviano se asienta en el principio de la mayoría con el reconocimiento y respeto de las minorías, para lo cual adopta un sistema electoral mixto que combina la representación proporcional y el criterio mayoritario para la elección de representantes".

De ahí que, en el establecimiento de circunscripciones electorales, ordena con base en el artículo 50 inciso c) que, para la elección de autoridades y representantes departamentales, se asignen escaños para la elección de representantes de las naciones y pueblos indígena originario campesinos que sean minoría poblacional en el departamento.

Para el caso de las autoridades y representantes de las autonomías regionales, se asignan escaños de elección directa para pueblos originarios que sean minoría poblacional en la región de acuerdo al Estatuto Autonómico Regional; igual situación se da para la elección de representantes municipales.

Para la Cámara de Diputados, se hace una división de los pueblos originarios a las circunscripciones especiales existentes, según el artículo 50 inciso c) de la ley electoral, y la distribución de los escaños será modificada por ley de la Asamblea Legislativa Plurinacional, después de un nuevo Censo Nacional de Población. Se establecen siete (7) Circunscripciones Especiales Indígena Originario Campesinas.

El artículo 61 de la referida ley establece que en cada circunscripción especial indígena originario campesina se elegirán un representante titular y su suplente, por simple mayoría de votos válidos y, en caso de empate, se dará lugar a una segunda vuelta electoral entre las candidatas o candidatos empatados.

Se establece que la postulación se efectuará a través de las organizaciones de naciones o pueblos indígena originario campesinos o las organizaciones políticas, debidamente registradas ante el Órgano Electoral Plurinacional, debiendo respetar criterios de paridad y alternancia. Finalmente, al igual que en el caso de Colombia, no se podrá votar simultáneamente en una circunscripción uninominal y en una circunscripción especial indígena originario campesina. 


\section{DERECHOELECTORAL}

Según los datos recabados del censo del 2012 por parte del Censo Nacional de Población y Vivienda del Instituto Nacional de Estadística (INE) de Bolivia, la población es 10059 856, de los cuales 5859879 (37\%) corresponden a población de naciones o pueblos minoritarios contemplados en la Ley del Régimen Electoral, como se presenta en la figura 2.

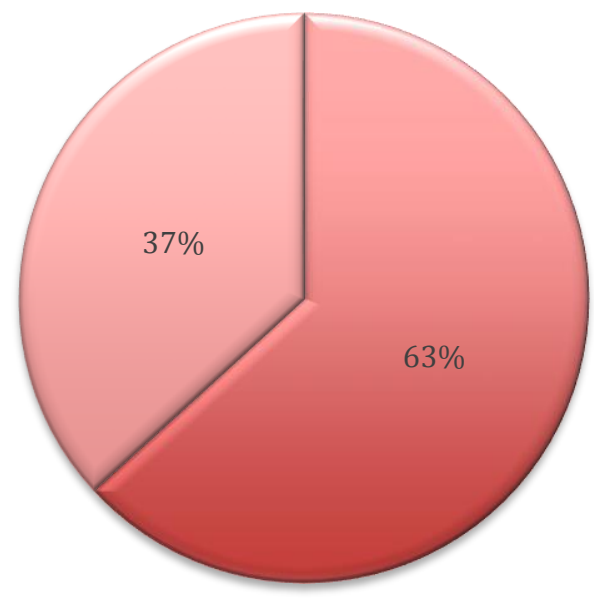

$\square$ Total población

๑Población indígena

Figura 2. Porcentaje de población a nivel nacional, Bolivia. Elaborado con base en los datos del censo del 2012 Instituto Nacional de Estadística.

La Cámara de Diputados de Bolivia se compone de 130 legisladores (Art. 146, Constitución Política), de los cuales y según la cantidad de población según censo del 2012, cada legislador representa a 77383 Bolivianos; en vista de que la Cámara de Diputados tiene siete miembros de representación indígena; para la cantidad de población indígena (5 859 879), debería corresponderle al menos 75 representantes de la Cámara. Esto quiere decir que los 7 diputados indígenas representan el 9,1\% de la población, cuando la población indígena corresponde a un $37 \%$, pese a ello, es la mayor cantidad de representación minoritaria en los países estudiados.

En opinión de Albó (2009), debe haber más circunscripciones especiales ahí donde haya más población indígena originaria minoritaria, lo cual contrasta las circunscripciones especiales con las demás uninominales por cuanto lo que busca es asegurar la presencia de pueblos tan minoritarios en la Asamblea Legislativa Plurinacional a pesar de su pequeño peso demográfico y su dispersión geográfica. 


\section{DERECHO EIECTORAL}

Considera que las circunscripciones especiales que abarcan a varios pueblos se deben acoplar para que varios se sientan representados por su diputado común y que este sea nombrado de alguna forma rotativa.

\section{c) Venezuela}

En el caso de Venezuela, la Constitución Política designa en su artículo 125 el derecho que tienen los pueblos indígenas a la participación política, garantizándose por parte del Estado representación en la Asamblea Nacional; ello se materializa con la asignación de tres diputados o diputadas y sus respectivos suplentes, de acuerdo con lo establecido en la ley electoral, respetando sus tradiciones y costumbres para los pueblos indígenas ${ }^{3}$. Cada diputado representa una región subdividida entre diversos grupos indígenas, las tres regiones conforman la circunscripción especial ${ }^{4}$.

Se establece en la misma Constitución las reglas que deben seguirse para la elección de los y las representantes indígenas a la Asamblea Nacional, donde la principal es el hablar su idioma indígena; además cumplir con alguno de los siguientes requisitos:

- Haber ejercido un cargo de autoridad tradicional en su respectiva comunidad.

- Tener conocida trayectoria en la lucha social en pro del reconocimiento de su identidad cultural.

- Haber realizado acciones en beneficio de los pueblos y comunidades indígenas.

- Pertenecer a una organización indígena legalmente constituida con un mínimo de tres años de funcionamiento.

La persona candidata ganará al obtener la mayoría de los votos válidos en su respectiva región o circunscripción. Los candidatos estarán en la boleta de su circunscripción y podrán ser elegidos por cualquier elector.

Por su parte, el artículo 5 del Reglamento a la Ley Orgánica de Procesos Electorales de Venezuela establece que el Consejo Nacional Electoral

\footnotetext{
${ }^{3}$ Artículo 182 de la Constitución Política de Venezuela.

${ }^{4}$ Artículo 137 de la Ley Orgánica de Procesos Electorales
} 


\section{DERECHO ELECTORAL}

procurará la elaboración de campañas divulgativas y educativas traducidas a los diferentes idiomas indígenas, para que sean transmitidas a través de emisoras de radio y televisoras comunitarias o locales que operen en los hábitats donde se encuentren asentadas las comunidades indígenas. Del mismo modo, se traducirán a los diferentes idiomas indígenas los materiales informativos, instructivos y formatos referentes a los procesos electorales.

La figura 3 reúne los datos recabados por el Instituto Nacional de Estadística para el censo del 2011; la población a nivel nacional correspondería a 28878 285, de los cuales 724592 (2,3\%) corresponden a población de indígena.

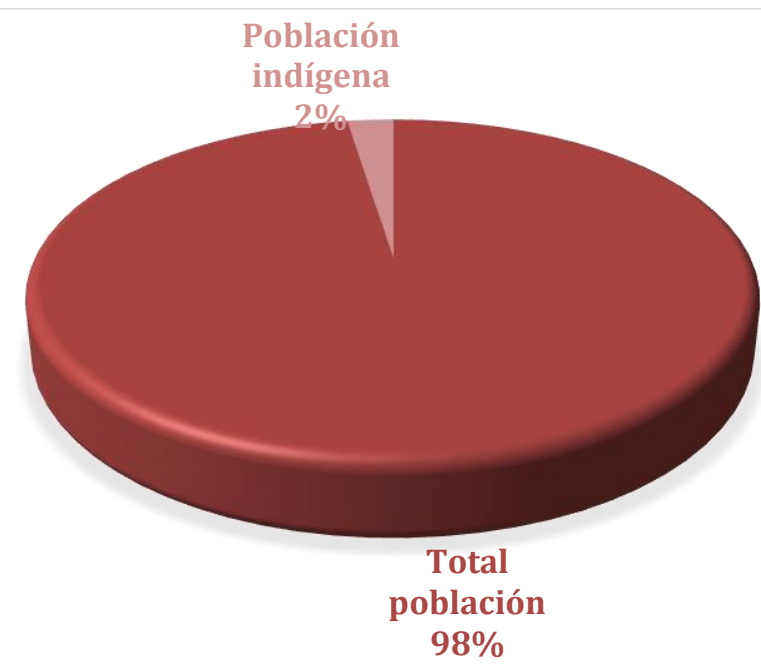

Figura 3. Porcentaje de población a nivel nacional, Venezuela. Elaborado con base en los datos del censo de 2011 Instituto Nacional de Estadística.

Dado que la elección de diputados se realiza con una base poblacional de uno coma uno por ciento de la totalidad del país, no hay un número exacto sobre el cual calcular la representación poblacional (Consejo Nacional Electoral, 2019); sin embargo, haciendo el ejercicio de dividir la totalidad de la población (28 878 285) entre el 1,1\% (317 661), tenemos que el porcentaje de diputaciones correspondería a 91 plazas. Esto quiere decir que los tres diputados indígenas representan el 2,7\% de la población; dado que la población indígena corresponde al 2,3\%, podemos concluir que, según el ejercicio realizado, la representación es proporcional a la población. 


\section{DERECHO EIECTORAL}

Lama la atención que, en el caso de Venezuela, pese a que tiene importantes regulaciones constitucionales y legales para los pueblos indígenas, no se hace referencia a otro tipo de etnia como, por ejemplo, la afrodescendiente.

\section{COMPARACIÓN Y CONCLUSIONES}

Ante el panorama en general, se hace eco de lo referido por Orozco (2010) en cuanto a que, en materia de participación política de minorías étnicas, los marcos normativos siguen siendo insuficientes para garantizar el acceso político a sectores que tradicionalmente han sido subrepresentados, al menos en forma proporcional a la cantidad de población según se.

Por eso, resulta de relevancia encontrar semejanzas y características similares entre los países estudiados, que permitan de alguna manera determinar los aspectos de mayor relevancia a ser analizados en futuras reformas de aplicación en países con propósitos de democratizar aún más la representación de minorías. Al respecto, la información recabada se resume en la tabla 1.

Tabla 1

Comparación de normativa legal y constitucional sobre representatividad de minorías étnicas. Colombia, Bolivia y Venezuela

\begin{tabular}{|c|c|c|c|}
\hline \multirow{2}{*}{ Normativa } & \multicolumn{3}{|c|}{ País } \\
\hline & COLOMBIA & BOLIVIA & VENEZUELA \\
\hline Constitución política & $\checkmark$ & $\checkmark$ & $\checkmark$ \\
\hline Ley especial & & $\checkmark$ & $\checkmark$ \\
\hline Circunscripción especial & $\checkmark$ & $\checkmark$ & $\checkmark$ \\
\hline Votación abierta & $\checkmark$ & $\checkmark$ & $\checkmark$ \\
\hline Requisitos de participación & $\checkmark$ & $\checkmark$ & $\checkmark$ \\
\hline $\begin{array}{l}\text { Cantidad de escaños fijos otorgados en } \\
\text { normativa }\end{array}$ & $4^{*}$ & 7 & 3 \\
\hline $\begin{array}{l}\text { Porcentaje población indígena respecto del } \\
\text { total }\end{array}$ & $4 \%$ & $37 \%$ & $2,3 \%$ \\
\hline $\begin{array}{l}\text { Porcentaje representación respecto del total } \\
\text { de la población }\end{array}$ & $1,72 \%$ & $9,1 \%$ & $2,7 \% * *$ \\
\hline
\end{tabular}

Nota: Elaboración propia de los datos obtenidos de la investigación.

(*) Solamente 1 es para indígenas.

$(* *)$ Según ejercicio de análisis de los datos obtenidos de la investigación. 


\section{DERECHO ELECTORAL}

Como primer elemento tenemos que, en común, los países estudiados cuentan con una asignación constitucional de la representación de minorías de forma expresa, ya sea con indicación de la cantidad de escaños destinados de manera fija, o bien de las circunscripciones electorales especiales.

En el caso de Colombia, se establecen cuatro escaños fijos para la Cámara de representantes, de los cuales solamente uno es para etnia indígena. En el caso de Bolivia, la cantidad de escaños para pueblos originarios o indígenas se encuentra ubicado en la Ley del Régimen Electoral; finalmente, para el caso de Venezuela, a pesar que la designación de escaños para la Cámara de Diputados se realiza en forma proporcional a la población, se establece en la Constitución Política un número fijo de tres representantes.

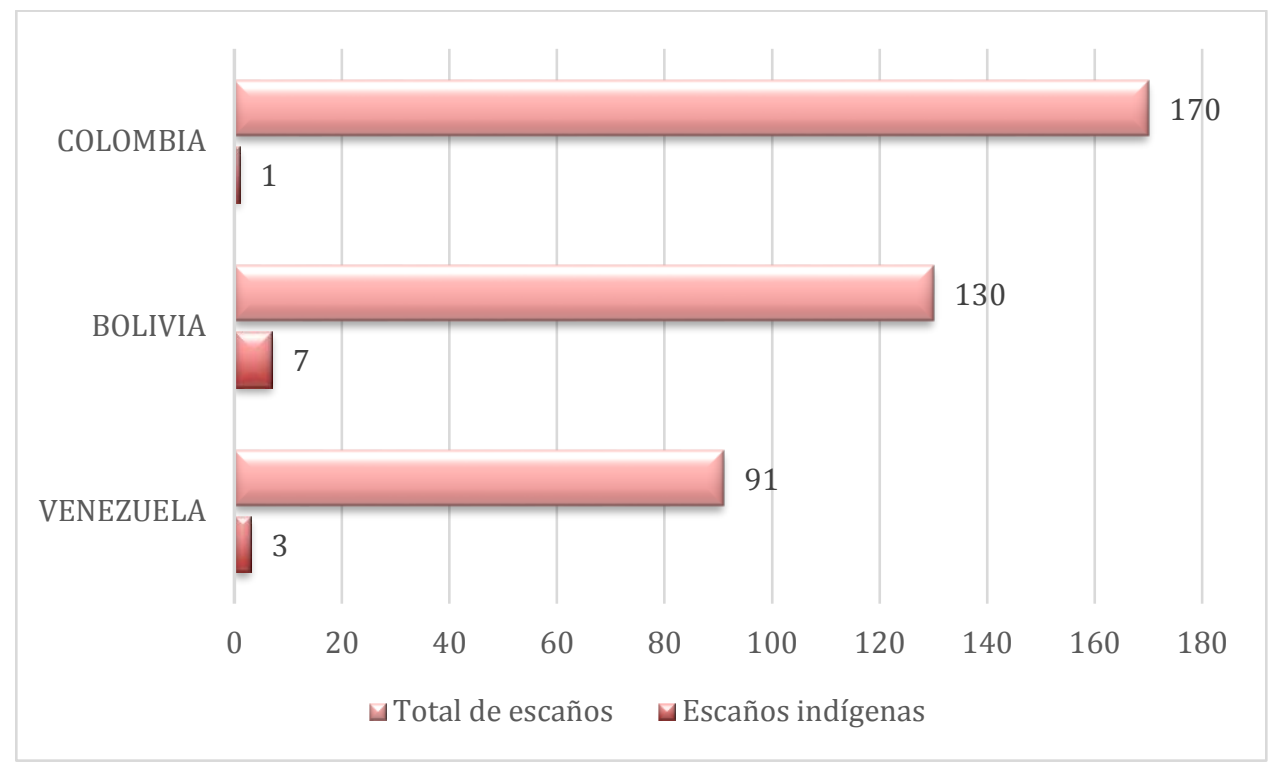

Figura 4. Comparación de circunscripciones electorales étnicas. Elaborado con base en los datos recopilados de diferentes fuentes durante la investigación.

En razón de lo anterior, las circunscripciones electorales étnicas se enfocan en la indígena (figura 4), con la excepción de Colombia cuya participación mayoritaria es la negra ( 2 escaños); si bien la circunscripción es especial para las etnias, se ven distribuidas en las áreas en las que se encuentran ubicadas estas, lo cual puede abarcar todo el país; en este aspecto, lo importante es lograr ubicar las regiones étnicas de relevancia, y sobre estas generar una circunscripción especial. En el caso de Venezuela y Bolivia, las regiones que conforman las circunscripciones están establecidas en la ley. Para el caso de Colombia, la circunscripción especial no está definida en una 


\section{DERECHO EIECTORAL}

parte específica del territorio, sino que cualquier persona puede votar por ella en la boleta de elección, por lo que puede ser elegido por cualquier persona a lo largo del país.

Como tercer elemento, si bien se establece una circunscripción especial, no se restringe el voto únicamente a las personas de determinada etnia, en cambio, cualquier persona que se ubique en el área de la circunscripción, puede votar; pese a ello, se aclara que, si bien la votación es abierta, en el caso de Colombia y Bolivia, se restringe a un único voto por circunscripción, ya que en dichos casos existe lista especial (étnica) y lista nacional (ordinaria). El votante solo puede votar en una u otra para lo cual se cuenta con 2 tipos de papeletas.

En cuanto a los requisitos de participación, en Colombia y Venezuela sí se limita quiénes pueden ser candidatos por dichas circunscripciones; la diferencia entre ambas radica en que la venezolana tiene una amplitud de circunstancias con la cual puede cumplir el requisito sin mecanismo de revisión, en cambio en la colombiana, se requiere un documento que acredite cumplir con el requisito y con ello la posibilidad de participar.

Finalmente, la asignación de escaños en el caso de Colombia y Venezuela está indicada en la Constitución; en el caso de Bolivia, se encuentra inferida en la ley, pero sin una asignación concreta; en cuanto a la diferencia entre cantidad de escaños, pese a que los países se encuentran en una similitud de circunstancias (tamaño, población, ubicación geográfica), de los datos recabados no se puede llegar a una conclusión del motivo de dicha diferencia.

De los datos recabados, vemos en la figura 5 que solamente en el caso de Venezuela la cantidad de escaños es proporcional a la representación de la población, con la salvedad de que los datos proporcionales fueron recabados de forma empírica al ser la designación de curules de forma proporcional a la población y, por ende, no tener un rubro fijo. 


\section{DERECHO ELECTORAL}

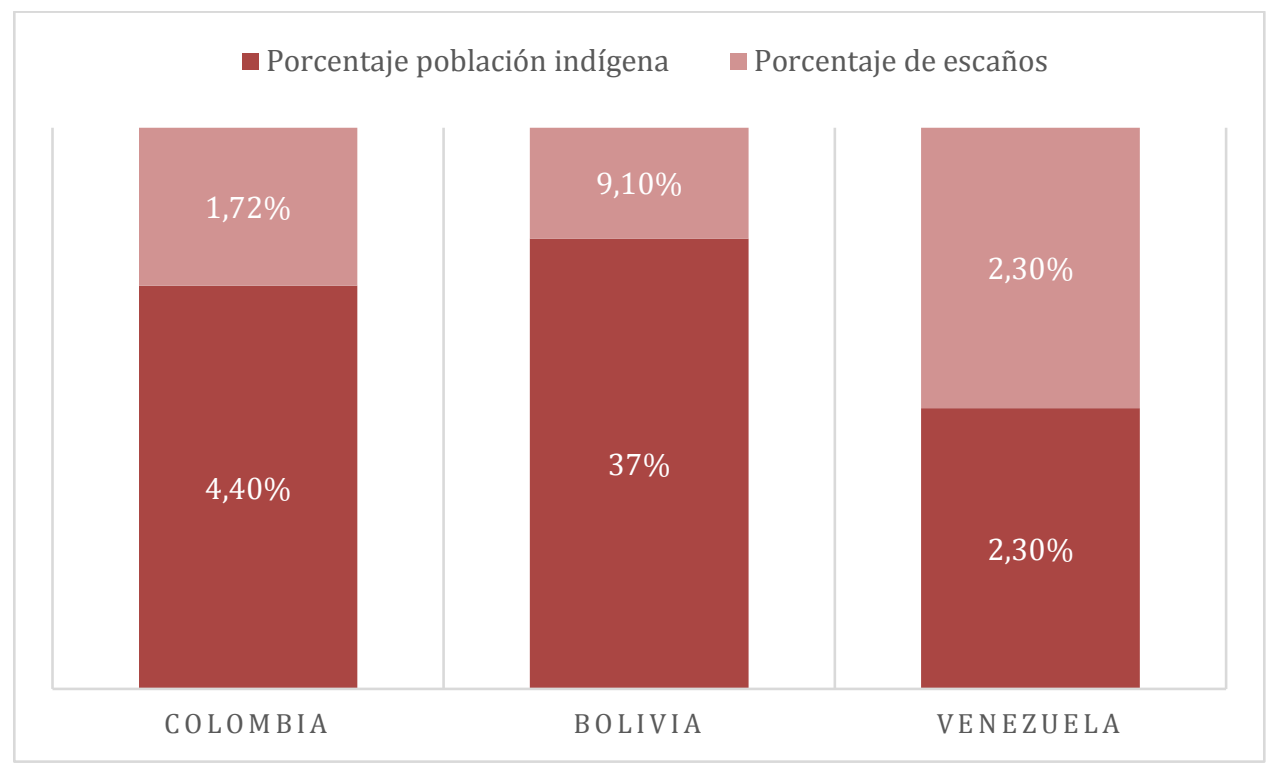

Figura 5. Comparación proporcionalidad de los escaños según la representación de la población. Elaborado con base en los datos recopilados de diferentes fuentes durante la investigación.

Pese a que la comparación se fundamentó en tres países únicamente, se realizó el estudio sobre otros seis países de Latinoamérica, los cuales no cuentan con ningún tipo de referencia a participación de minorías, y mucho menos enfocada en minorías étnicas.

En el caso de Panamá 5 , Paraguay ${ }^{6}$, Perú ${ }^{7}$ y $\mathrm{Chile}^{8}$, pese a que cuentan con grupos minoritarios como indígenas y afrodescendientes, ni la Constitución Política, ni las leyes electorales o de organización electoral hacen referencia alguna a circunscripciones especiales dirigidas a dichos grupos (Fuentes, 2018); la referencia a representatividad de minorías lo es en el mecanismo electoral que utilizan por medio de la fórmula de D'hondt, para garantizar la representación de minorías. Ello; sin embargo, favorece, aunque no garantiza, la participación grupos políticos minoritarios, mas no así de grupos sociales como los del caso de estudio.

\footnotetext{
${ }^{5}$ Ver el Diagnóstico de la Población Indígena de Panamá con base en los Censos de Población y Vivienda de 2010 (https://www.contraloria.gob.pa/inec/archivos/P6571INDIGENA_FINAL_FINAL.pdf).

6 Ver: Pueblos indígenas en el Paraguay. Resultados finales de población y viviendas 2012 (https://www.dgeec.gov.py/Publicaciones/Biblioteca/indigena2012/Pueblos\%20indigenas\%20en\%20el\%20Para guay\%20Resultados\%20Finales\%20de\%20Poblacion\%20y\%20Viviendas\%202012.pdf).

7 Ver: Base de pueblos indígenas u originarios (https://bdpi.cultura.gob.pe/).

${ }^{8}$ Ver: Base de datos de Pueblos Indígenas u Originarios (https://www.ine.cl/estadisticas/sociales/etnias).
} 


\section{DERECHO ELECTORAL}

En Ecuador, pese a que la población indígena representa casi el $45 \%$ de la población, según la Confederación de Nacionalidades Indígenas del Ecuador (CONAIE), no se ve proporcionalmente reflejada ni en la representación parlamentaria ni en los puestos de gobierno, pese a su reconocimiento formal en la Constitución Política del país (Bello, 2001); similar situación se da en el caso de Guatemala.

En todo caso, se ve reflejado el esfuerzo por garantizar la representación de minorías étnicas mediante mecanismos que permitan el acceso a quienes realmente los representen, y de forma tal que se ajuste al sistema electoral de cada país

\section{REFERENCIAS BIBLIOGRÁFICAS}

Albó, X. (Abril, 2009). Las circunscripciones especiales indígenas. Boletín Virtual, 8(279). Recuperado de https://www.alainet.org/es/active/29867

Alto Comisionado para los Derechos Humanos (ACNUDH). (2010). Derechos de las minorías: Normas internacionales y orientaciones para su aplicación. Recuperado de https://www.ohchr.org/Documents/Publications/MinorityRights_sp.pdf

Barth, F. (1976). Los Grupos étnicos y sus fronteras. Recuperado de https://museo-etnografico.com/pdf/puntodefuga/151209barth.pdf

Bello, A. (2001). Discriminación étnico-racial y xenofobia en América Latina y el Caribe. Serie Política Sociales $n .^{\circ}$ 47. Chile: CEPAL. Recuperado de https://repositorio.cepal.org/bitstream/handle/11362/5987/1/S01050412_e s.pdf

Bolivia (2009). Constitución Política del 07 de febrero.

Bolivia (2010). Ley del Régimen Electoral n. ${ }^{\circ} 026$ del 30 de junio.

Bolivia. Instituto Nacional de Estadística (INE) de Bolivia. Censo Nacional De Población y Vivienda-2012. Recuperado de http://datos.ine.gob.bo/binbol/RpWebEngine.exe/Portal?BASE=CPV2012CO M\&lang $=\mathrm{ESP}$

Chile. Instituto Nacional de Estadísticas (2002). Estadísticas sociales de los pueblos indígenas en Chile. Recuperado de https://www.ine.cl/estadisticas /sociales/etnias 


\section{DERECHO EIECTORAL}

Colombia Congreso de la República (2019). Cámara de Representantes. Recuperado de http://www.camara.gov.co/index.php/la-camara

Colombia (1991). Constitución Política del 04 de julio.

Colombia. Corte Constitucional (2010). Sentencia C-702/10. Demanda de inconstitucionalidad de acto legislativo por vicios de procedimiento/demanda de inconstitucionalidad contra acto legislativo

Colombia (2001). Ley 649 del 27 de marzo. Reglamenta el artículo 176 de la Constitución Política.

Colombia. Departamento Administrativo Nacional de Estadísticas (DANE) (2018). Resultados del Censo Nacional de Población y Vivienda 2018-Pueblos Indígenas. Recuperado de https://www.dane.gov.co/index.php/estadisticaspor-tema/demografia-y-poblacion/grupos-etnicos

Colombia. Registraduría Nacional del Estado Civil (s.f.). Representación, participación y curules especiales de minorías étnicas. Recuperado de https://www.registraduria.gov.co/IMG/pdf/Representacion_Politica_de_las_ minorias_en_Colombia_UR.pdf

Colombia. Universidad del Rosario (2019). Comunidad Raizal. Recuperado de https://www.urosario.edu.co/jurisprudencia/catedra-vivaintercultural/ur/Comunidades-Etnicas-de-Colombia/Comunidad-Raizal/

Colprensa (8 marzo, 2018) ¿Qué son las circunscripciones especiales indígena y afro en el Congreso? Colombia. Radio Nacional de Colombia. Recuperado de https://www.radionacional.co/noticia/actualidad/que-son-las-

circunscripciones-especiales-indigena-afro-congreso

Corona, R. (Jul.-Set., 2006). Minorías y grupos diferenciados: claves para una aproximación conceptual desde la perspectiva internacional. Ius Revista Jurídica Digital, (22). Recuperado de http://www.unla.mx/iusunla22/reflexion/minorias\%20y\%20grupos\%20difer nciados.htm\#_ftn1

Flores, C. (2014). Sistemas electorales y minorías étnicas en la Europa central y del este: de la transición a la consolidación de la democracia. Revista de Estudios Políticos, (166, 41-67. Recuperado de https://dialnet.unirioja.es/servlet/articulo?codigo $=4934189$

Fuentes C. y Sánchez M. (2018). Asientos reservados para pueblos indígenas. Serie Policy Papers n. ${ }^{\circ} 1$. Recuperado de https://www.ciir.cl/ciir_2019/wpcontent/uploads/2018/07/policy-paper-UPP-n\%C2\%BA1-2018-1.pdf 


\section{DERECHOEIECTORAL}

Herrán, O. (Jul.-Dic., 2009). Las Minorias Étnicas Colombianas en la Constitución Política de 1991. Prolegómenos. Derechos y Valores, 12(24), 189-212.

Orozco, J. (Ene.-Jun., 2010). Las reformas electorales en perspectiva comparada en América Latina. Revista de Derecho Electoral, (9), 1-32. Recuperado de http://www.tse.go.cr/revista/art/9/orozco_henriquez.pdf.

Panamá (20190). Diagnóstico de la Población Indígena de Panamá con base en los Censos de Población y Vivienda de 2010. Recuperado de https://www.contraloria.gob.pa/inec/archivos/P6571INDIGENA_FINAL_FINA L.pdf

Paraguay. Dirección General de Estadística, Encuestas y Censos (2012). Pueblos indígenas en el Paraguay. Resultados finales de población y viviendas 2012. Recuperado de https://www.dgeec.gov.py/Publicaciones/Biblioteca/ indigena2012/Pueblos\%20indigenas\%20en\%20el\%20Paraguay\%20Resulta dos\%20Finales\%20de\%20Poblacion\%20y\%20Viviendas\%202012.pdf

Perú (2019). Base de datos de Pueblos Indígenas u Originarios. Recuperado de https://bdpi.cultura.gob.pe/

Pinedo, E. (Jul.-Dic., 2010). Las cuotas de participación electoral en Perú. Características y algunos resultados. Revista Digital de Derecho Electoral, (10), 1-33. Recuperado de http://www.tse.go.cr/revista/art/10/pinedo_bravo.pdf

Venezuela (1999). Constitución Política el 30 de diciembre.

Venezuela (2009). Ley Orgánica de Procesos Electorales del 31 de julio.

Venezuela (2013). Reglamento General de la Ley Orgánica de Procesos Electorales del 18 de enero.

Venezuela. Consejo Nacional Electoral (2019). Cargos diputados a la Asamblea Nacional. Recuperado de http://www4.cne.gob.ve/web/sistema_electoral/cargos/diputados_an.php

Venezuela. Instituto Nacional de Estadística (2011). La población indígena de Venezuela, Censo 2011. Recuperado de http://www.ine.gov.ve/documentos/SEN/menuSEN/pdf/subcomitedemografi $\mathrm{ca} /$ Indigena/BoletinPoblacionIndigena.pdf 